\title{
Modified Double-Lavered Flap Technique for Closure of an Oroantral Fistula: Surgical Procedure and Case Report ${ }^{+}$
}

\author{
Garibaldi Joseph, Grasso Sara *, Piazzai Matteo, Merlini Alessandro and Del Buono Caterina \\ U.O. Odontostomatologia, Galliera Hospital Genoa, Mura delle Cappuccine, 14, 16128 Genova, Italy; \\ jgaribaldi@libero.it (G.J.); piazzai@libero.it (P.M.); alemerlo91@hotmail.it (M.A.); caterina.db@libero.it (D.B.C.) \\ * Correspondence: mini.sgrasso@gmail.com; Tel.: +39-3347010533 \\ + Presented at the XV National and III International Congress of the Italian Society of Oral Pathology and \\ Medicine (SIPMO), Bari, Italy, 17-19 October 2019.
}

Published: 12 December 2019

The formation of an oro-antral communication following avulsion of the lateral and posterior teeth of the maxilla is not an exceptional event in dental practice; it can undergo spontaneous resolution or the formation of a fistula that requires surgical treatment in order to create an absolutely hermetic barrier between the oral environment and the maxillary sinus [1]. The aim of the study was to provide a review of the literature on the surgical techniques currently in use for the resolution of oro-antral communications, that are the trapezoidal, rotated vestibular, rotated palatine, buccal fat pad and double-layered flap techiniques; therefore to describe the central theme of the study that is the technique of mucogingival plastic surgery called modified double-layered flap techinque moderately invasive and less subject to medium and long term recurrences [2].

We performed a review of the methods used to solve small oroantral communications (Figure 1) and carried out a case report on the alternative technique proposed by the Odontostomatology Unit of the Galliera Hospital in Genoa, ie the modified double-layered flap technique.

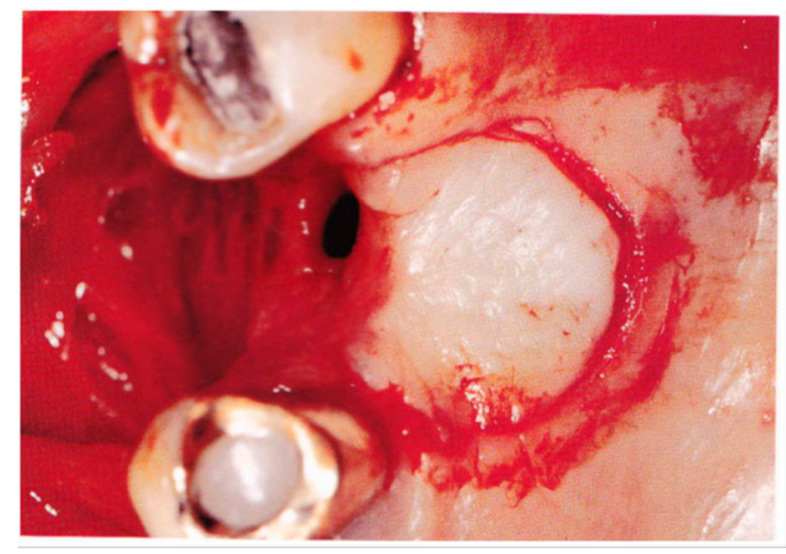

Figure 1. Example of a small oro-antral communication treated with the technique proposed.

The intent therefore remains to propose a valid protocol that is not a substitute but an alternative to the pre-existing ones, which have already been exhaustively described in the literature. The present protocol (Figure 2) has also been recognized and published by the authoritative source of the British Journal of Oral and Maxillofacial Surgery. 


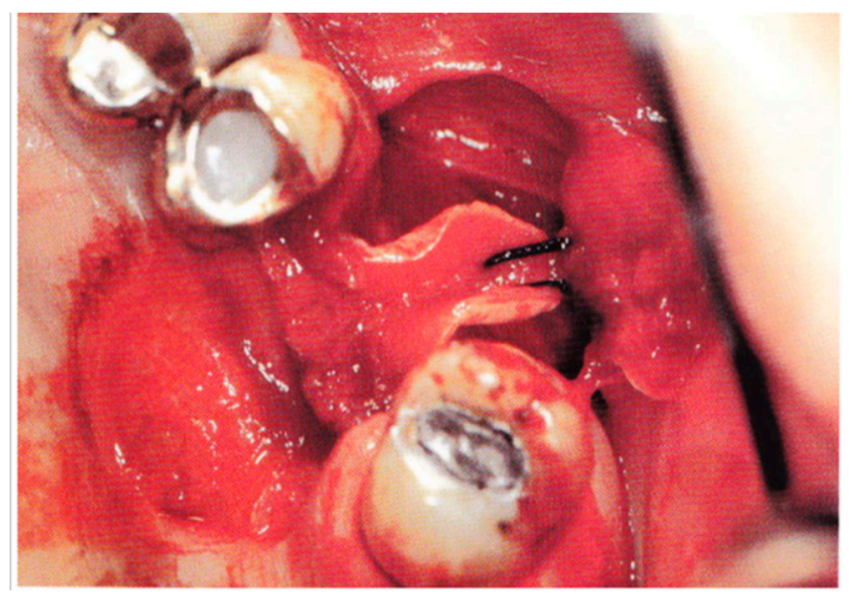

Figure 2. U-shaped anchorage to the vestibular fornix: the palatal flap constitutes the inner lining and the displacement of the vestibular flap optimally closes the primitive defect.

With regards to the satisfactory results obtained, we can state that this method is predictable.

The oro-sinus and in particular the alveolus-sinus communications are mostly sequelae of previous dental treatments and avulsions. The dentist is required to diagnose and identify the most appropriate therapeutic approach. Among the various techniques available, the modified doublelayered flap technique is certainly a valid choice, as it has good predictability [3].

Conflicts of Interest: The authors declare no conflict of interest.

\section{References}

1. Abuabara, A.; Cortez, L.V.; Passeri, L.A.; de Moraes, M.; Moreira, R.W. Evaluation of different treatments for oroantral/oronasal communications: Experience of 112 cases. Int. J. Oral Maxillofac. Surg. 2006, 35, 155-158.

2. Adams, T.; Taud, D.; Rosen, M. Repair of oroantral communications by use of a combined surgical approach: Functional endoscopic surgery and buccal advancement flap/buccal fat pad graft. J. Oral Maxillofac. Surg. 2015, 73, 1452-1456.

3. Felisati, G.; Chiapasco, M.; Lozza, P.; Saibene, A.M.; Pipolo, C.; Zaniboni, M.; Biglioli, F.; Borloni, R. Sinonasal complications resulting from dental treatment: outcome-oriented proposal of classification and surgical protocol. Am. J. Rhinol. Allergy 2013, 27, e101-e106.

(C) 2019 by the authors. Licensee MDPI, Basel, Switzerland. This article is an open access article distributed under the terms and conditions of the Creative Commons Attribution (CC BY) license (http://creativecommons.org/licenses/by/4.0/). 